\title{
分析计算机技术在办公自动化中的应用探究
}

于晓丽

DOI:10.32629/ems.v2i2.708

[摘 要] 现阶段我国已经进入到信息时代,计算机技术水平不断提升,也被广泛普及和使用。就目前情况来看,我国很 多企业都将计算机技术使用在办公中,这就让企业内部的办公已经变为自动化,在提升工作效率的同时将企业经济效 益不断增加。正因为这样,才应该将计算机技术有效使用,让其在办公中发挥作用,促进企业发展。本文就计算机技术 在办公自动化中应用的问题和计算机技术应用特征展开分析,提出计算机技术在办公自动化中的应用,希望可以对相 关人员有所帮助。

[关键词] 计算机技术; 办公自动化; 问题; 应用

在办公室系统中应用的最关键技术就是计算机技 术,计算机技术让办公自动化不断完善。办公自动化不光 可以帮助企业节省很多进行管理的时候还可以将员工 的工作效率和质量提升, 现在已经被很多企业和单位接 受和认可。但是计算技术在我国办公自动化中应用还存 在一些问题, 这些问题会阻碍计算机技术的作用发挥和 企业经济效益的提升。所以现阶段应该找出这些问题进 行具体分析和研究,从而让计算机技术发挥其全部作用, 提高工作效率,促进企业的发展。

\section{1 计算机技术在办公自动化中应用所出现的问题}

1.1 工作人员没有较高的专业素质

计算机技术在这几年间快速发展, 让办公自动化也 得到了不断的发展。但是我国大多数企业都是中小型的, 没有充足的工作人员。现有的工作人员大多数对计算机 技术也没有一个全面的了解和掌握, 这就会出现不能将 计算机技术的作用有效发挥的现象 ${ }^{[1]}$ 。反之, 正因为计算 机技术的加人, 让这些工作人员的工作量加大,一些工作 人员没有较高的专业素质和技术水平, 很容易在使用计 算机技术的时候不小心将企业信息泄露或者是出现错 误。这些情况对于企业的发展来说非常不利。不难看出, 工作人员需要具有较高的专业素质, 并对计算机技术有 一个全面的了解才可以让办公自动化正常的运行和发 展。

的完工。所以, 建筑单位一定要通过合理的奖罚制度来制 约工程参与人员行为。

\section{5 结论}

由于建筑工程涉及内容较多, 其自身综合性较强, 而 且施工过程中的任何一些细小环节都将影响到最终的 工程质量, 且施工技术存在纰漏也会直接影响质量。因 此, 要想保证工程施工质量, 首先要明确技术, 然后制定严 格的监管制度, 运用于每一个环节中。施工技术与施工管 理相配合, 同进行, 实现其有效融合, 从而保证工程质量,

\section{2 没有较高的办公室管理质量}

想要让办公自动化有一个良好的运行, 就需要对其 进行有效管理, 将企业制定的管理制度当做办公自动化 运行的辅助工具, 从而促进企业发展。但是部分企业还没 有认识到办公自动化对自身发展的意义, 企业和管理人 员之间没有良好的配合，在使用计算机技术的时候知识 一种形式,这样就无法对进行有效的管理和控制,会让管 理也变得形式化。导致这些问题出现的主要原因就是企 业没有将办公自动化深人到每一个工作环节当中, 这不 仅会出现浪费计算机技术功能的现象, 还会影响企业的 发展和经济效益的提升。

\section{3 没有良好的办公自动化运行环境}

在办公自动化中应用计算机技术, 需要为其提供一 个良好的环境, 这个环境对于办公自动化的运行来说也 同样重要 ${ }^{[2]}$ 。但是, 我国有一些企业并没有重视办公自动 化, 这就会导致在应用计算机技术的时候没有一个良好 的环境。其实, 在办公自动化中应用计算机技术主要是为 了将工作人员的工作效率和质量提升。但是正因为没有 良好的环境会让应用的效果出现反差, 可能会出现混淆 工作职能或者是模糊不清等情况, 从而让企业内部的员 工将工作效率降低,无法为企业带来更多的经济效益。所 以,在办公自动化中应用计算机技术的时候,应该创建一 个良好的运行环境, 这不光可以让计算机技术的功能发

推进建筑行业稳步发展。

[参考文献]

[1]柴成栋. 建筑工程施工技术及现场施工管理措施 分析[J].建筑技术开发,2019,46(13):67+68.

[2]潘荣华. 现阶段建筑工程施工技术及其现场施工 管理分析[J].建材与装饰,2016(14):235 + 236.

[3]陈绍森.建筑工程施工技术及其现场施工管理探 讨[J].城市建筑,2019,16(12):163+ 164. 
挥出来, 还可以让企业在发展的过程中增加更多的经济 效益。

\section{2 办公自动化中计算机技术的应用特点}

2.1 可以单独处理数据

办公自动化两个主要部分就是计算机应用程序还 有登录到数据库进行数据查询, 但是这两部分内容都是 独立的, 并没有任何的联系。与此同时这两个部分还不会 互相影响, 这也正是计算机技术为办公自动化带来的优 势。工作人员在使用的时候需要注意, 计算机应用程序和 数据库在有不同的逻辑结构, 不会出现干扰的情况, 不必 担心。

\section{2 具有完整性}

在办公自动化中应用计算机技术会出现三种不同 的特征, 那就是: 数据程序在处理的时候具有一致性; 数 据信息具有准确性; 数据信息的完成性。每一位工作人 员使用相同账号登录办公软件的时候, 会出现统一的数 据库, 这就是数据的一致性; 在工作人员使用数据库的时 候, 输人的数据和数据库中原有的数据是相同类型, 这就 是正确性;工作人员在使用数据库的时候数据库中的数 据自己不会出现丢失的情况, 并保持数据完整,这就是所 谓的数据信息完整性。

\section{3 具有安全性}

对于一个企业来说, 企业有非常多的重要信息不能 泄露,所以就需要保证办公自动化信息的安全。在一般情 况下所使用的手段就是安全防护, 像是保护重要的文件 或者是将重要数据和其他数据分开等一些方法。还可以 对工作人员经常登录的办公室软件的账号、权限以及口 令等进行监测。防止数据丢失还可以设置自动上传到云 端,方便丢失后查找。

\section{4 加密管理}

在企业的计算机中会储存较为庞大的自动化办公 运行数据, 其中还可能包括企业的机密文件和数据, 为了 可以让这些机密数据得到保障, 管理计算机数据库的人 员应该使用计算机中的加密技术对这些数据进行加密。 可以使用加密程序、办公加密软件还有设置密码或者是 口令等, 避免在数据库受到不法人员人侵的时候导致企 业丢失非常重要的机密文件。

\section{3 计算机技术在办公自动化中的应用}

\section{1 文字和数据处理技术}

就目前情况来看, 我国各行各业的企业都在快速发 展, 大多数企业都已经将计算机技术应用到办公自动化 当中,能够对企业的大量数据信息进行整理和处理。也正 因为如此, 计算机技术中的文字和数据处理技术才可以 在办公自动化中得到良好的发展。

\section{1 .1 应用数据处理技术}

现如今我国经济不断发展, 这就让很多企业的管理
文章类型: 论文 | 刊号( ISSN):2705-0637(P)/2705-0645(O)

层人员还有决策人员需要掌握和企业发展相关的信息 和资料,从而进行定量分析,研究出适合企业长远发展的 策略,而计算机数据处理技术可以帮助其完成操作 ${ }^{[3]}$ 。这 项技术常见的办公软件就是 office 软件, 可以对相关人 员所给出的数据进行整理、加工、储存等一系列的操作, 最主要的是使用的时候非常方便。并且还可以使用和表 格相关的软件, 更加直观的看到和企业有关的一些数据 信息, 也可以将所想要看到的数据信息使用图表的形式 展现出来。

\subsection{2 应用文字处理技术}

我国现阶段已经进人了信息时代，很多信息技术出 现在人们的面前。对于文字来说,早已经从手写变为了印 刷的方式, 最后演变成了今天的计算机文字。主要原因 是,计算机技术在输人文字的时候有较高的准确性,所以 将文字处理技术应用到企业的日常办公中已经是一场 非常平常的事情。并且这项技术在公司管理还有处理业 务等方面都有一定的地位,也成为不能缺少的办公工具。 其实,对文字进行处理知识计算机技术的一个简单操作， 员工还可以使用文字软件对输人到计算机中的文字进 行排版和整理,让文字看上去非常美观整洁。除此之外, 还可以将不小心删除的文字信息利用历史记录找回来, 这样一来就可以防止文字丢失, 将员工的工作效率提升 上去。

\section{2 应用多媒体技术}

在传统的信息交流媒体当中，只能将信息单向的并 且被动传播出去, 而使用多媒体技术可以让人们主动选 择和控制想要传播和观看的信息。多媒体技术之所以深 受企业的喜爱主要有两点原因:第一,综合性较强。多媒 体技术能够将视频、声音、图片等多种表现形式融为一 体; 第二,多媒体可以让人和机器之间互相交流。除此之 外,多媒体技术还是信息载体,具有多样化和交互性等特 点。

办公自动化想到得到发展肯定离不开多媒体技术。 在办公中总会使用图片、视频等一些工具,如果将多媒体 技术的作用有效发挥,让其帮助工作人员将语音、视频还 有图片等进行处理,可以满足许多工作人员的工作要求。 此外,这项技术还能够提升办公信息处理的应用价值。

最重要的一点是, 在很多企业的办公中都已经将多 媒体融入进去, 像是工作人员已经制作好了介绍产品或 者是汇报工作的演示文稿,可以使用投影仪进行演示,让 其余工作人员更加清晰的看到演示文稿的内容, 也让演 示文稿提升了许多感染力。利用多媒体演示文稿可以让 图形、声音还有视频等多种形式表现出来,让介绍和汇报 变得非常生动, 内容也随之丰富起来, 帮助演示人员吸引 观看人员的注意力。也正是应用了多媒体技术还有数字 影像技术,才可以让办公设备变得更加现代化,从而构建 


\section{建筑防水材料防霉性能检测分析}

辛海洋 刘方浩

山东北方创信防水科技集团股份有限公司天津分公司

DOI:10.32629/ems.v2i2.709

[摘 要] 建筑工程中,防水系统直接关系到建筑物的正常使用。由于防水材料终日处于阴暗潮湿的环境中, 因此较易 产生大量的霉菌, 不仅会影响到建筑物的安全及防水性能, 并且会对人们的身体健康产生严重的危害, 加强建筑防水 材料的防䨢性能检测已成为一项重要工作。本文首先介绍了国内外防䨢性能检测发展现状, 并分析了建筑防水材料 防雫性能检测方法, 最后对检测结果进行了评判。

[关键词] 建筑防水材料; 防䨢性能;检测分析

建筑防水卷材和密封胶产品是建筑体中不可或缺 的基础性材料,尤其在地下室、欴房、卫生间中,可防止水 流渗漏到建筑物结构中, 从而影响到建筑物的正常使用。 由于此类空间潮湿度较高, 为霉菌的大量繁殖营造出了 适宜的环境, 霉菌会腐蚀生长基层, 导致出现一定的危 害。厨卫间为保持密封性会应用到密封胶,如密封胶表面 长期存在霉菌, 会逐渐发黄、发黑, 人体若接触此类材料, 可影响到呼吸系统的健康。另外,位于种植屋面的防水材 料如产生大量的霉菌, 可造成植物长势不佳, 并造成屋顶 的防水材料受到破坏, 严重时会造成屋顶渗漏甚至塌陷 的情况, 因此必须对此予以重视。由此可见,建筑物中的 防水材料如出现霉菌, 会影响到建筑物的正常使用。针对

了新型办公自动化系统。工作人员也不要在面对超级多 的文件, 只需要使用一台电脑和多媒体设备就可以轻松 的完成办公,将工作效率和质量提升。

\section{3 应用网络技术}

计算机技术中的网络技术是办公自动化中非常重 要的一部分内容。现阶段我国各企业在使用计算机技术 的时候已经打破空间和时间的限制, 可以随时随地的办 公,也将信息共享实现 ${ }^{[4]}$ 。让办公人员可以对所需要进行 处理的信息实时处理,提高工作效率。除此之外,工作人 员还可以更加全面的搜集、整理和处理信息, 在遇到问题 的时候能够及时的找到解决方案, 从而实现科学管理。

在办公自动化中, 主要将网络技术应用在以下几个 方面:第一,电子邮件的应用。每一个公司和部门都有着 某种联系, 使用电子邮件可以让每个部门之间将信息快 速的传递,不用害怕延误传递信息时间,将处理信息的能 力和效率不断提升; 第二,收集信息。在全球经济一体化 的背景下, 网络技术加人到办公自动化中, 可以让办公效 率有效提升。工作人员在任何时间和地点都可以在网络 上搜集到自己想要的信息和知识, 也可以使用电话会议 随时随地的进行交谈,促进办公自动化的发展。
此种情况, 我们可通过开展建筑防水材料防霉性能检测 的方法, 认识到需菌的生长情况, 并将结果交由治理人员 加以改善。

\section{1 国内外防霉性能检测发展现状}

人类的生产及生活均离不开各类建筑物, 但由于建 筑物中较易滋生大量的需菌, 故应研究霉菌治理的方法。 防需性能检测最早出现在上世纪 40 年代,由美国霍普金 斯大学创建了世界上第一家需菌实验室。从事此领域研 究的人员在大量的工作中, 制定出了霉菌检测的标准。经 过长期的实践,此项技术逐渐传递到世界各地,各个国家 依据自身情况, 制定出了适合本地特点的检测标准及方 法。日本于 1953 年制定并通过了《耐霉性能试验方法》,

\section{4 结束语}

总而言之,想要让办公自动化持续稳定发展,肯定不 能离开计算机技术。计算机技术在办公自动化中的应用 能够将员工的工作效率和质量不断提升, 并且减少办公 错误的出现, 提高工作准确率。需要注意的是, 计算机技 术的种类非常多，应该将不同种类的技术使用在不同办 公领域中,从而发挥其作用,促进企业的发展和经济效益 的提升。

\section{[参考文献]}

[1]董航.计算机技术在办公自动化中的应用探究 [J].科技创新导报,2018,15(35):137+139.

[2]胡金荣.分析办公自动化中的计算机技术应用 [J].电脑乐园・信息化教学,2018(8):329.

[3]韩云飞.计算机技术在办公自动化中的应用分析 [J].无线互联科技,2018,15(15):135+136.

[4]杨志强.计算机办公自动化技术分析及应用探讨 [J]. 时代经贸,2019,473(12):100+101.

\section{作者简介}

姓名: 于晓丽; 性别: 女; 民族: 汉; 籍贯: 山东; 身份 证号:370682198910063148 